\title{
Unifying Paradigms of Quantum Refrigeration: A Universal and Attainable Bound on Cooling
}

Clivaz, Fabien; Silva, Ralph; Haack, Geraldine; Brask, Jonatan Bohr; Brunner, Nicolas; Huber, Marcus

Published in:

Physical Review Letters

Link to article, DOI:

10.1103/physrevlett.123.170605

Publication date:

2019

Document Version

Publisher's PDF, also known as Version of record

Link back to DTU Orbit

Citation (APA):

Clivaz, F., Silva, R., Haack, G., Brask, J. B., Brunner, N., \& Huber, M. (2019). Unifying Paradigms of Quantum Refrigeration: A Universal and Attainable Bound on Cooling. Physical Review Letters, 123(17), [170605]. https://doi.org/10.1103/physrevlett.123.170605

\section{General rights}

Copyright and moral rights for the publications made accessible in the public portal are retained by the authors and/or other copyright owners and it is a condition of accessing publications that users recognise and abide by the legal requirements associated with these rights.

- Users may download and print one copy of any publication from the public portal for the purpose of private study or research.

- You may not further distribute the material or use it for any profit-making activity or commercial gain

- You may freely distribute the URL identifying the publication in the public portal 


\title{
Unifying Paradigms of Quantum Refrigeration: A Universal and Attainable Bound on Cooling
}

\author{
Fabien Clivaz, ${ }^{1,2}$ Ralph Silva, ${ }^{1,3}$ Géraldine Haack, ${ }^{1}$ Jonatan Bohr Brask, ${ }^{1,4}$ Nicolas Brunner, ${ }^{1}$ and Marcus Huber $\odot^{2}$ \\ ${ }^{1}$ Department of Applied Physics, University of Geneva, 1211 Geneva 4, Switzerland \\ ${ }^{2}$ Institute for Quantum Optics and Quantum Information (IQOQI), \\ Austrian Academy of Sciences, Boltzmanngasse 3, A-1090 Vienna, Austria \\ ${ }^{3}$ Institute for Theoretical Physics, ETH Zürich, 8093 Zürich, Switzerland \\ ${ }^{4}$ Department of Physics, Technical University of Denmark, Fysikvej, Kongens Lyngby 2800, Denmark
}

(Received 1 April 2019; revised manuscript received 24 September 2019; published 22 October 2019)

Cooling quantum systems is arguably one of the most important thermodynamic tasks connected to modern quantum technologies and an interesting question from a foundational perspective. It is thus of no surprise that many different theoretical cooling schemes have been proposed, differing in the assumed control paradigm and complexity, and operating either in a single cycle or in steady state limits. Working out bounds on quantum cooling has since been a highly context dependent task with multiple answers, with no general result that holds independent of assumptions. In this Letter we derive a universal bound for cooling quantum systems in the limit of infinite cycles (or steady state regimes) that is valid for any control paradigm and machine size. The bound only depends on a single parameter of the refrigerator and is theoretically attainable in all control paradigms. For qubit targets we prove that this bound is achievable in a single cycle and by autonomous machines.

DOI: 10.1103/PhysRevLett.123.170605

Characterizing the ultimate performance limits of quantum thermal machines is directly related to the understanding of energy exchanges at the quantum scale, and hence to the formulation of thermodynamic laws valid in the quantum regime [1]. A central challenge towards this goal is to identify the relevance of different levels of control that can be achieved over quantum mechanical machines and how this determines the thermodynamic limits in the quantum regime. To analytically derive such fundamental limits, it is instructive to observe that the time evolution of closed quantum systems is unitary in quantum mechanics. Discussing nontrivial changes of energy and entropy on a target system thus requires a conceptual separation of a thermodynamic process into a target system, upon which a desired task is performed, and a "machine," comprised of several other quantum systems, which is used to perform the task upon the target. The total evolution will be a global unitary operation and feature a limited depth or complexity (i.e., machines and the baths' constituents they couple to have a finite size) and can either be assumed to be energy changing, if one allows for a coherent control of the process, or alternatively energy preserving in an incoherent control paradigm. Furthermore, machines can operate in a cyclic manner, such that each of these unitary operations can be repeated an arbitrary number of times. To derive self-contained and nontrivial bounds on thermodynamic performance, the state of the machine for each of the repetitions should be thermal with respect to its Hamiltonian. Additionally, to include heat engines, part of the machine could be thermalized with respect to a higher temperature. All these different paradigms are illustrated in Fig. 1, and include paradigmatic scenarios, such as autonomous quantum thermal machines [2-4], heat bath algorithmic cooling [5-10], and quantum otto engines $[11,12]$ for finite size processes. Our framework is a special case of the generalized framework exposed in Ref. [13] but differs from any of the case studies made there. In the limit

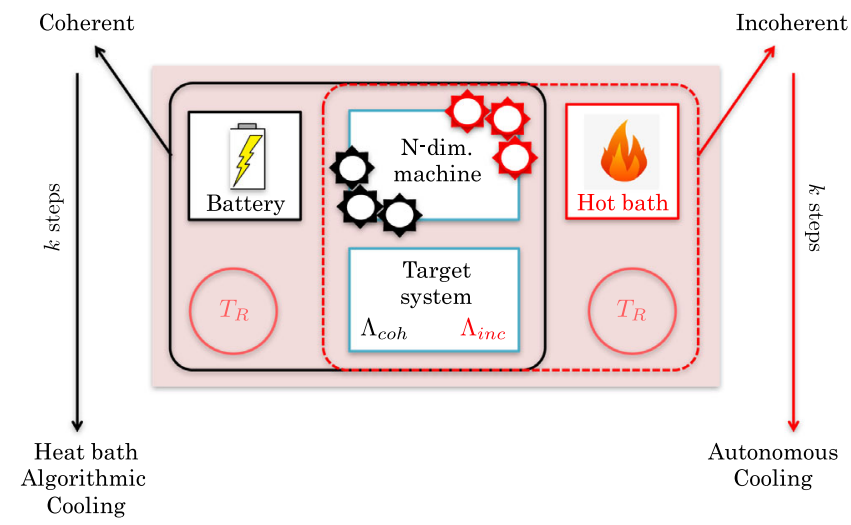

FIG. 1. Schematic representation of the paradigms of coherent and incoherent control for quantum refrigeration. For a target qubit, the coldest state of the infinite cycle regime of the incoherent paradigm is the steady state achieved by autonomous cooling. For a target qubit and a product qubit machine, the coherent paradigm is a special instance of heat bath algorithmic cooling with no compression qubit. 
of infinite size, the limiting cases are the resource theory of thermodynamics [14-17] for incoherent operations and for coherent control general completely positive and trace preserving maps, that make cooling to the ground state trivially possible, emphasizing the role of complexity in the third law of thermodynamics [18-22].

A task of paramount importance for quantum technologies is refrigeration and, depending on the paradigm, different limitations have been derived for specific machine designs. Indeed, it seems a daunting task to derive bounds beyond specific, low complexity machines, as the potential Hamiltonians, dimensions, and number of repetitions present an overwhelming amount of parameters to optimize over. Thermodynamics as a theory, however, has been astoundingly successful in deriving general statements that are independent of the microscopic complexity, by identifying a small number of relevant parameters that ultimately determine the limits of processing energy.

We should first clarify that cooling a quantum system can have several meanings. For a system initially in a thermal state, one can drive it to a thermal state of lower temperature. Alternatively, since for some paradigms fixing the entire spectrum is actually too strong a condition, one could just consider increasing its ground-state population or its purity, or decreasing its entropy or its energy (see, e.g., discussions on passivity [23-26]). These notions are in general nonequivalent for target systems of arbitrary dimension and determining the fundamental limits to cooling is therefore dependent on the choice of target function. However, by using majorization theory, we are able to derive results that hold for all of the abovementioned notions of cooling.

Setting.-We consider a target system of dimension $d_{S}$ with Hamiltonian $H_{S}=\sum_{i=0}^{d_{S}-1} E_{i}\left|E_{i}\right\rangle_{S}\left\langle E_{i}\right|$, where $E_{i} \leq E_{j}$ for $i<j$ and a thermodynamic machine of finite size $d_{M}$ with Hamiltonian $H_{M}=\sum_{i=0}^{d_{M}-1} \mathcal{E}_{i}\left|\mathcal{E}_{i}\right\rangle_{M}\left\langle\mathcal{E}_{i}\right|$, where $\mathcal{E}_{i} \leq \mathcal{E}_{j}$ for $i<j$, both surrounded by a thermal bath at temperature $T_{R}$ (or inverse temperature $\beta_{R}$ ). Without loss of generality we assume $E_{0}=\mathcal{E}_{0}=0$. We also write $\mathcal{E}_{\max }=\mathcal{E}_{d_{M}-1}$ and for a qubit target, $d_{S}=2, E_{S}=E_{1}$. The joint initial state of target and machine is given by $\rho_{\mathrm{SM}}=\tau_{S}\left(\beta_{R}\right) \otimes \tau_{M}\left(\beta_{R}\right)$, where $\tau\left(\beta_{R}\right)$ denotes a thermal state at inverse temperature $\beta_{R}$. Our goal is to cool the target system. After the application of a single unitary the target state is changed as $\operatorname{Tr}_{M}\left[U \rho_{\mathrm{SM}} U^{\dagger}\right]=: \Lambda\left[\tau_{S}\left(\beta_{R}\right)\right]=: \rho_{S}^{\prime}$. To induce a nontrivial change of the target system, out-of-equilibrium resources have to be used, which we conceptually divide into two extremal cases: Either we use external coherent control to induce any energy changing unitary of target and machine (see, e.g., Ref. [27]), inducing the map $\Lambda_{\text {coh }}$ on the system. Or we consider an energetically closed system, i.e., consider unitaries that commute with the total system-machine Hamiltonian $H=H_{S}+H_{M}$, inducing $\Lambda_{\text {inc }}$ on the target. This application of a unitary represents a single cycle of the machine. Between cycles the machine is rethermalized, where individual machine components can thermalize either with the environment at temperature $T_{R}$ or draw further resources from a hot bath at temperature $T_{H}$. For many cycles we denote the number of cycles $k$ by a superscript on the map $\Lambda_{\mathrm{coh} / \mathrm{inc}}^{k}$. Of particular interest to us is the unbounded cycle regime, i.e., as $k \rightarrow \infty$, which we denote by $p_{0}^{*}$. The setting is visually represented in Fig. 1 and the asymptotic relations of the induced maps is summarized in Table I in the Supplemental Material [28].

Results. - In the following we consider an arbitrary sequence of operations and prove a bound that holds for any control paradigm and any machine size in the limit of infinite cycles. This bound only depends on the maximal energy gap of the machine, irrespective of the structure of $H_{M}$. The bound can be attained for both coherent and incoherent machines. Finally, for qubit targets, the bound can even be obtained in a single cycle by the smallest possible coherent machine (i.e., a single qubit machine), as well as via the smallest autonomous refrigerator (i.e., a two-qubit machine).

The energetics of quantum systems only depend on the diagonal elements in the energy eigenbasis, which through unitary evolution change unistochastically. This already gives a recipe for single cycle cooling that is analytically optimal in terms of majorization theory: The global diagonal of the joint target and machine density matrix of the final state is always majorized by the initial diagonal (Shur-Horn Lemma). Thus, the optimal unitary is one that puts all the largest eigenvalues in the diagonal entries that contribute to the ground state population of the target, the next largest in those entries contributing to the first excited state of the target, and so on. Which unitary that is, depends on all energy gaps and respective eigenvalues for each cycle.

Descriptions of thermodynamic machines, however, should go beyond such optimal single cycles and also allow for a repetition of cycles to determine its ultimate limits. Ideally, each cycle can be perfectly separated and the machine rethermalized in between cycles. In both coherent and incoherent paradigms we thus also consider the limit of infinite repetitions of optimal unitary operations, where the machine can be perfectly rethermalized in between. While optimal unitary operations can at least in principle be described for every cycle of arbitrary machine and target systems, they will in general depend on the entire spectrum and do not identify relevant machine parameters for determining performance. Indeed, determining simple and universal bounds on reachable target temperatures and revealing the relevant parameters of machines in all paradigms, even after infinite cycle repetitions, is the main result of this Letter. We present four theorems gauging the cooling performances for the different types of machines, prove universal upper bounds on cooling, and demonstrate their respective attainability. In the accompanying article [29], we furthermore investigate the work cost of achieving these bounds. 
Universal bound on cooling.-Unless stated otherwise, we consider an arbitrary machine, i.e., any spectrum and size. It turns out, however, that no matter the microscopic physics of the machine, only a single machine parameter determines the ultimate cooling bound, namely, the largest energy gap $\mathcal{E}_{\text {max }}$.

Theorem 1: (Universal bound on cooling) For any machine and control paradigm, in the limit of infinite cycles, i) for a qubit target, the ground state population is upper bounded by

$$
p_{0}^{*}=\frac{1}{1+e^{-\beta_{R} \mathcal{E}_{\max }}},
$$

(ii) for an arbitrary target of dimension $d_{S}$, the vector of eigenvalues of the final state is majorized by that of the following state,

$$
\rho_{S}^{*}=\frac{1}{\mathcal{Z}} \sum_{n=0}^{d_{S}-1}\left(e^{-\beta_{R} \mathcal{E}_{\max }}\right)^{n}\left|E_{n}\right\rangle_{S}\left\langle E_{n}\right|,
$$

as long as the initial state is majorized by $\rho_{S}^{*}$. In particular, the ground state population, purity, entropy, and average energy of the final state are bounded by those of $\rho_{S}^{*}$.

Before we discuss the proof, we would like to make a few remarks. The qubit bound, first derived in Ref. [30], and also appearing in Ref. [31] corresponds to an inverse temperature of

$$
\beta^{*}=\frac{\beta_{R} \mathcal{E}_{\max }}{E_{S}} .
$$

The crucial parameter is the ratio between the populations of the excited and ground state, $g=e^{-\beta_{R} \mathcal{E}_{\max }}$. The bound (2) for higher dimensional systems has the same property, that the ratio of populations for every pair of successive energy levels is given by $g$.

We emphasize the advantage of a bound based on majorization. Since the state $\rho_{S}^{*}$ is the unique passive state [32] that majorizes all others also attainable by coherent operations, it upper (lower) bounds every Schur convex (concave) function of the eigenvalues, which includes the various notions of cooling listed in the theorem.

Proof of Theorem 1.-First, for machines using coherent operations, using the Schur-Horn Lemma for the joint state of target and machine $\rho_{\mathrm{SM}}$, one can verify (see the Supplemental Material [28]) that the system after a single cycle operation $\rho_{S}^{\prime}$ satisfies $\rho_{S}^{\prime} \prec \rho_{S}^{*}$ as long as $\rho_{S} \prec \rho_{S}^{*}$. By induction, one arrives at $\rho_{S}^{(n)} \prec \rho_{S}^{*}$ for all $n$.

Furthermore, note that a hotter thermal state is always majorized by a colder thermal state and majorization is stable under tensor products, see Corollary 1.2. of Ref. [33]. Thus, having access to a hot thermal bath to rethermalize our machine before a cycle, or part of it, if the machine has a tensor product structure, will only enable us to reach states that are majorized by the initial state. Thus a hot thermal bath does not allow us to reach a state that cannot already be reached by a coherent resource, completing the proof.

Attaining the cooling bound.-The bound defined in Eq. (2) only depends on the subspace of the machine with the "coldest" ratio of populations. Indeed, one can construct an explicit cooling protocol as follows: consider the following simple joint unitary operation between target and machine,

$$
\begin{aligned}
U_{i}= & \mathbb{1}-\left|E_{i-1} \mathcal{E}_{\max }\right\rangle\left\langle E_{i-1} \mathcal{E}_{\max }|-| E_{i} \mathcal{E}_{0}\right\rangle\left\langle E_{i} \mathcal{E}_{0}\right| \\
& +\left|E_{i-1} \mathcal{E}_{\max }\right\rangle\left\langle E_{i} \mathcal{E}_{0}|+| E_{i} \mathcal{E}_{0}\right\rangle\left\langle E_{i-1} \mathcal{E}_{\max }\right| .
\end{aligned}
$$

Each $U_{i}$ is a simple qubit swap, between the $i$ th pair of successive energy eigenstates of the target, and the $\mathcal{E}_{\max }$ subspace of the machine, and will lead to the population of the lower energy state $p_{i-1}$ increasing by some $\Delta_{i}$ (this may be negative). We define the "coherent max-swap" operation as the one that performs the $U_{i}$ corresponding to the greatest positive value of $\Delta_{i}$ (if none exists, then no unitary is performed). The choice and implementation of $U_{i}$ is preceded and followed by a unitary on the target that leaves the target in a passive state.

Theorem 2: (Coherent attainability) The state of the target under the repeated application of either one of the coherent max-swap operation or the optimal coherent operation converges to $\rho_{S}^{*}$.

Proof.-Since the state of the target is passive before a particular $U_{i}$ is performed, and $\Delta_{i}>0$ in this case, the population is always moved from a smaller eigenvalue to a larger one. Thus the final state always majorizes the initial one. This is also true for the optimal coherent operation, since by construction it leaves the target in a state that majorizes all others. Thus the ordered partial sums of eigenvalues of the target under repeated application of either operation form monotonically increasing sequences. As these sums are bounded by one, both protocols must converge.

In the Supplemental Material [28], we show that the state converged to is a fixed point of the coherent max-swap operation, and that all fixed points of this protocol have the property of majorizing $\rho_{S}^{*}$. By Theorem 1 , the final state under any protocol must be majorized by $\rho_{S}^{*}$, proving that the only possible convergent point of the max swap is $\rho_{S}^{*}$ itself.

The proof for the case of optimal coherent operations follows from the fact that the state under many cycles majorizes the state under an equal number of cycles of any other coherent operations (see Supplemental Material [28]). Thus the state converged to must majorize that of the max swap, but also still be majorized by $\rho_{S}^{*}$ (Theorem 1). Therefore it also converges to $\rho_{S}^{*}$.

This shows that the universal bound is tight in the coherent case. Since the operations involved are nonenergy 
conserving, one cannot conclude the same for the incoherent paradigm. Nonetheless, a minor addition to the machine enables a similar statement.

In the coherent case we only required to swap qubit subspaces in the target with the maximum energy gap of the machine. Each of these swaps can be made energy preserving by adding one more qubit to the machine to bridge the energy difference, and thermalizing this qubit to a hot temperature $T_{H}>T_{R}$ in between cycles. This defines an incoherent version of the max-swap protocol.

Theorem 3: (Incoherent attainability)In the limit $T_{H} \rightarrow \infty$, one can incoherently cool the target to at least the coherent cooling bound of $\rho_{S}^{*}$ in the infinite cycle limit, if one extends the machine by (at most) $d_{S}-1$ qubits, each with energy gap $\mathcal{E}_{\text {max }}-\left(E_{i}-E_{i-1}\right), i \in\left\{1,2, \ldots, d_{S}-1\right\}$, or more generally, any extension that has qubit subspaces with these energy gaps [34].

Proof.-The proof is analogous to that of Theorem 2. Here too, the (incoherent) max-swap operation has the property of the final state always majorizing the initial one. It follows that the repeated application converges. One can prove (see Supplemental Material [28]) that the convergent point is a fixed point of the operation, and that in the limit $T_{H} \rightarrow \infty$, the fixed points all majorize $\rho_{S}^{*}$, proving attainability as desired.

Theorem 3 shows that the two paradigms are in fact closely related to one another in terms of cooling performance. In the limit of $T_{H} \rightarrow \infty$, a finite resource of the same dimension of the target suffices to bridge the gap between them.

Bridging extremal cooling paradigms.-So far, we dealt exclusively with stroke type machines, in the sense that we allowed for rethermalization or unitary operations in well separated discrete time steps. One may, however, wonder if the bounds on cooling set by Theorems 1-3 are also valid for autonomous thermal machines, where thermalization and machine cycles happen simultaneously and continuously. Interestingly, a link exists between those machines and our incoherent paradigm, see also Ref. [35]. In essence, the energy-preserving unitaries of the incoherent paradigm are replaced by a time-independent energy-preserving interaction Hamiltonian between the target system and the machine. It is then straightforward to prove the following statement.

Theorem 4: (Autonomous machines correspondence) Consider an arbitrary machine and a qubit target system. When the target coupling to the thermal bath is zero, one can cool the target to $\beta^{*}$ with an autonomous machine by extending the machine as in Theorem 3.

Proof.-This follows directly from known results for small autonomous machines. Following the derivation in [36], which is based on a linear master equation, one can replace the second qubit by the subnormalized qubit $\rho_{0, \max }=$ $(1 / \mathcal{Z})\left(|0\rangle\left\langle 0\left|+e^{-\beta_{R} \mathcal{E}_{\max }}\right| \mathcal{E}_{\max }\right\rangle\left\langle\mathcal{E}_{\max }\right|\right)$. Furthermore, adding energy levels to the machine between $|0\rangle$ and $\left|\mathcal{E}_{\max }\right\rangle$ does not affect the analysis since the added levels remain invariant under this evolution.

While it is expected that the correspondence holds for higher dimensional targets as well, it requires an analysis of the master equation that we leave for further work. The zero coupling of the target to the environment needed for exact correspondence comes from the fact that in the repeated cycles paradigm, there is never any reheating between the cycles, which corresponds to an open quantum system in which the target is not coupled to a bath. Any realistic coupling would only worsen the bound. The correspondence of Theorem 4 links extremal paradigms of control in quantum thermal machines, perfect batteries and welltimed operations on the one hand, and autonomous machines with no external source of work or timing control on the other, demonstrating that increased control on a quantum system does, after all, not significantly impact the cooling performance in this context, but that the difference manifests as different work costs and the actual challenge rather lies in designing appropriate interaction Hamiltonians for autonomous machines [37].

Smallest machines for maximal cooling.-Interestingly the smallest possible implementation can already attain the bounds. For a qubit target of energy gap $E_{S}$, the simplest coherent machine consists of a single qubit, of energy gap $E_{M}$. The Hilbert space of the joint target and machine system is spanned by $\left\{|i j\rangle_{\mathrm{SM}}\right\}_{i, j=0,1}$. Maximal cooling is achieved by a single unitary operation, swapping the states $|01\rangle_{\text {SM }}$ and $|10\rangle_{\text {SM }}$. The final state of the target thus has the same populations and Gibbs ratio as the initial state of the machine, i.e.,

$$
\beta^{*} E_{S}=\beta_{R} E_{M},
$$

which matches Eq. (3) for maximal cooling with $E_{M}=\mathcal{E}_{\max }$. This single qubit machine is also sufficient to cool higher dimensional targets to the bound (2), using the coherent max-swap protocol (Theorem 2).

Note that if the machine had additional energy levels in between $|0\rangle_{M}$ and $|1\rangle_{M}$, then a single swap would not be sufficient to recover Eq. (5) for a qubit target, and one would only do so in the limit of infinite cycles. Furthermore, higher dimensional targets would also be cooled at a slower rate (per cycle). In this sense, a single qubit machine is more effective than a more complex machine. The advantage of a more complex machine is revealed when analyzing the work cost of the operation (see Ref. [29]), and in general can help increase $\mathcal{E}_{\max }$ by composition.

In the incoherent paradigm, a single qubit machine is unable to cool. The simplest machine allowing for cooling features an extra qubit of energy $E_{A}=E_{M}-E_{S}$, as proposed in Theorem 3. This corresponds to the smallest autonomous refrigerator [2]. The optimal unitary for cooling is now the swap between the states $|010\rangle_{S M A}$ and $|101\rangle_{S M A}$. Comparing this to the single qubit coherent machine, we see that the swap is identical with respect to 
the target $(S)$ and the original machine $(M)$, and the role of the additional qubit $(A)$ is to enable this transition for incoherent cooling by bridging the energy gap between $S$ and $M$. In coherent cooling, this role is implicitly fulfilled by the battery that allows for arbitrary unitary operations.

Cooling over multiple cycles consists in repeatedly thermalizing $M$ to $T_{R}$ and $A$ to $T_{H}$ and performing the relevant swap. In the limit of infinite cycles, the temperature achieved by the target is given by

$$
\beta_{\text {inc }}^{*} E_{S}=\beta_{R} E_{M}-\beta_{H}\left(E_{M}-E_{S}\right) .
$$

In the limit of $T_{H} \rightarrow \infty$, the second term on the right-hand side vanishes and we get Theorem 3 .

Conclusion.-We derive universal and attainable bounds for cooling using any quantum machine and target system. Interestingly, this bound only depends on the largest energy gap present within the quantum refrigerator and is independent of all other spectral properties and valid for all temperature regimes. For qubits, this bound is already attainable by the simplest possible machines: either by a single cycle of a coherent one-qubit machine or, in the continuous autonomous quantum refrigerator paradigm, as a steady state of a two-qubit machine. These results unify different operational approaches to quantum thermodynamics and thus go beyond one particular approach. They embody one of the central conceptual pillars of statistical physics, that, despite the potential complexity, thermodynamic tasks can be characterized by a small number of system parameters that need no detailed knowledge of the microstates. The universal bounds and the attainability protocols presented here, are, in general, of course highly idealized and go beyond realistic control over many-body quantum systems. That makes the attainability by few qubit machines all the more interesting, however, as they could potentially be realized with state-of-the-art quantum technologies. A more detailed analysis of few qubit machines in both paradigms, including a finite number of cycles and the respective work costs of achieving the temperature bound can be found in Ref. [29]. Future investigations should include a trade-off between complexity and achievable $\mathcal{E}_{\text {max }}$, as the required unitaries would quickly become impossible to even approximate in the regime of large machines. Another question beyond asymptotics is the actual convergence rate. We showcase the simplest case in the Supplemental Material [28] and hope to gain more indepth insight in the future. One could also further extend the operational paradigm by including quantum measurements of the target or working fluid, such as in Ref. [38], keeping in mind that there one also implicitly assumes large measurement machines that replace the refrigerator [39]. Or one could investigate the advantage of more general thermal couplings, such as in Ref. [40]. Finally, in the cooling task considered here, both initial and final states are diagonal in the energy eigenbasis, such that further limitations from the manipulation of coherences do not apply $[41,42]$. It could be interesting to study how initial coherences affect the results.

We are grateful to F. Hirsch, P. P. Hofer, M.-O. Renou, and T. Krivachy for fruitful discussions. We would also like to acknowledge all referees for productive and challenging comments. Several authors acknowledge the support from the Swiss NFS (National Science Foundation) and the NCCR QSIT: R. S. through the Grants No. 200021_169002 and No. 200020_165843, N. B. through the Starting grant DIAQ and Grant No. 200021_169002, F. C. through the AMBIZIONE Grant No. PZ00P2_161351, and G. H. through the PRIMA Grant No. PR00P2_179748 and Marie-Heim Vögtlin Grant No. 164466. M. H. acknowledges support from the Austrian Science Fund (FWF) through the START Project No. Y879-N27. J.B.B. acknowledges support from the Independent Research Fund Denmark.

[1] J. Goold, M. Huber, A. Riera, L. del Rio, and P. Skrzypczyk, J. Phys. A 49, 143001 (2016).

[2] N. Linden, S. Popescu, and P. Skrzypczyk, Phys. Rev. Lett. 105, 130401 (2010).

[3] A. Levy and R. Kosloff, Phys. Rev. Lett. 108, 070604 (2012).

[4] N. Brunner, N. Linden, S. Popescu, and P. Skrzypczyk, Phys. Rev. E 85, 051117 (2012).

[5] L. J. Schulman and U. Vazirani, in Proc. 31'st ACM STOC (Symp. Theory of Computing) (1999), pp. 322-329.

[6] P. O. Boykin, T. Mor, V. Roychowdhury, F. Vatan, and R. Vrijen, Proc. Natl. Acad. Sci. U.S.A. 99, 3388 (2002).

[7] D. K. Park, N. A. Rodriguez-Briones, G. Feng, R. Rahimi, J. Baugh, and R. Laflamme, Heat bath algorithmic cooling with spins: Review and prospects, in Electron Spin Resonance (ESR) Based Quantum Computing (Springer, New York, 2016), pp. 227-255.

[8] N. A. Rodriguez-Briones, E. Martin-Martinez, A. Kempf, and R. Laflamme, Phys. Rev. Lett. 119, 050502 (2017).

[9] S. Raeisi and M. Mosca, Phys. Rev. Lett. 114, 100404 (2015).

[10] N. A. Rodriguez-Briones and R. Laflamme, Phys. Rev. Lett. 116, 170501 (2016).

[11] W. Niedenzu, D. Gelbwaser-Klimovsky, A. G. Kofman, and G. Kurizki, New J. Phys. 18, 083012 (2016).

[12] W. Niedenzu, V. Mukherjee, A. Ghosh, A. G. Kofman, and G. Kurizki, Nat. Commun. 9, 165 (2018).

[13] A. M. Alhambra, M. Lostaglio, and C. Perry, Quantum 3, 188 (2019).

[14] F. G. S. L. Brandao, M. Horodecki, J. Oppenheim, J. M. Renes, and R. W. Spekkens, Phys. Rev. Lett. 111, 250404 (2013).

[15] G. Gour, M. P. Müller, V. Narasimhachar, R. W. Spekkens, and N. Y. Halpern, Phys. Rep. 583, 1 (2015).

[16] R. Gallego, J. Eisert, and H. Wilming, New J. Phys. 18, 103017 (2016).

[17] M. P. Müller, Phys. Rev. X 8, 041051 (2018). 
[18] L. Masanes and J. Oppenheim, Nat. Commun. 8, 14538 (2017).

[19] H. Wilming and R. Gallego, Phys. Rev. X 7, 041033 (2017).

[20] J. G. Richens, A. M. Alhambra, and L. Masanes, Phys. Rev. E 97, 062132 (2018).

[21] J. Scharlau and M. P. Mueller, Quantum 2, 54 (2018).

[22] F. Ticozzi and L. Viola, Sci. Rep. 4, 5192 (2014).

[23] W. Pusz and S. L. Woronowicz, Commun. Math. Phys. 58, 273 (1978).

[24] R. Alicki and M. Fannes, Phys. Rev. E 87, 042123 (2013).

[25] K. V. Hovhannisyan, M. Perarnau-Llobet, M. Huber, and A. Acin, Phys. Rev. Lett. 111, 240401 (2013).

[26] M. Perarnau-Llobet, K. V. Hovhannisyan, M. Huber, P. Skrzypczyk, J. Tura, and A. Acin, Phys. Rev. E 92, 042147 (2015).

[27] J. Aberg, Phys. Rev. Lett. 113, 150402 (2014).

[28] See Supplemental Material at http://link.aps.org/ supplemental/10.1103/PhysRevLett.123.170605 for a detailed exposition of the proofs.

[29] F. Clivaz, R. Silva, G. Haack, J. B. Brask, N. Brunner, and M. Huber, companion paper, Phys. Rev. E 100, 042130 (2019).

[30] A. E. Allahverdyan, K. V. Hovhannisyan, D. Janzing, and G. Mahler, Phys. Rev. E 84, 041109 (2011).

[31] D. Reeb and M. M. Wolf, New J. Phys. 16, 103011 (2014).
[32] Diagonal in the energy eigenbasis, with eigenvalues arranged in decreasing order with respect to increasing energy.

[33] J. V. Bondar, Linear Algebra Appl. 360, 1 (2003).

[34] By extending the machine $M$ with $M_{1}$ we mean that the initial state of the machine is now $\rho^{M} \otimes \rho^{M_{1}}$, where both $\rho^{M}$ and $\rho^{M_{1}}$ are thermal. The total Hamiltonian is hence $H=H_{S}+H_{M}+H_{M_{1}}$, and again unitaries commuting with $H$ are considered.

[35] R. Uzdin, A. Levy, and R. Kosloff, Phys. Rev. X 5, 031044 (2015).

[36] P. Skrzypczyk, N. Brunner, N. Linden, and S. Popescu, J. Phys. A 44, 492002 (2011).

[37] M. T. Mitchison, M. Huber, J. Prior, M. P. Woods, and M. B. Plenio, Quantum Sci. Technol. 1, 015001 (2016).

[38] L. Buffoni, A. Solfanelli, P. Verrucchi, A. Cuccoli, and M. Campisi, Phys. Rev. Lett. 122, 070603 (2019).

[39] Y. Guryanova, N. Friis, and M. Huber, arXiv:1805 .11899.

[40] N. A. Rodrguez-Briones, J. Li, X. Peng, T. Mor, Y. Weinstein, and R. Laflamme, New J. Phys. 19, 113047 (2017).

[41] P. Ćwikliński, M. Studziński, M. Horodecki, and J. Oppenheim, Phys. Rev. Lett. 115, 210403 (2015).

[42] A. Streltsov, G. Adesso, and M. B. Plenio, Rev. Mod. Phys. 89, 041003 (2017). 